\title{
A Medium Viscous Acrylic Cement Enhances Uniformity of Cement Filling and Reduces Leakage in Cancellous Bone Augmentation
}

\author{
Andreas Boger and Kurtis Wheeler \\ RÆD Biomaterials, Synthes GmbH, Eimattstrasse 3, 4436 Oberdorf, Switzerland \\ Correspondence should be addressed to Andreas Boger, boger.andreas@synthes.com
}

Received 18 April 2011; Accepted 15 May 2011

Academic Editors: R. N. Barbosa and C. Derail

Copyright $\odot 2011$ A. Boger and K. Wheeler. This is an open access article distributed under the Creative Commons Attribution License, which permits unrestricted use, distribution, and reproduction in any medium, provided the original work is properly cited.

\begin{abstract}
Vertebroplasty is an efficient procedure to treat osteoporotic vertebral fractures. Cement leakage is considered to be the most severe complication during vertebroplasty. At high cement viscosities, the risk of leakage is reduced. However, injection forces are highly increased, requiring the use of high force injection systems with the lack in tactile force feedback. Therefore, an acrylic cement was developed showing medium initial viscosity and which still allows to be manually applied using syringes. An established model favoring leakage was used to observe the filling pattern and leaked cement mass. The method used included the simulation of body temperature, and all materials submitted are approved medical products. When applied just after preparation, the medium viscous cement resulted in good cement filling and less cement leakage than low viscosity cement. The added clinical value of the new cement is that the waiting time for the physician will be shortened to zero minutes without compromising the safety for the procedure.
\end{abstract}

\section{Introduction}

In the last 10 years, percutaneous vertebroplasty has become one of the most important techniques in the treatment of weakened or collapsed vertebrae and therefore an indispensable tool in the fight against pain induced by osteoporosis and other conditions [1]. Percutaneous vertebroplasty leads to immediate pain relief in $80 \%$ to $90 \%$ of patients $[1,2]$. However, bone cement leakage is a common occurrence, and the associated risks represent the main complication for vertebroplasty [3]. Leakage can occur through a path caused by irregularities in the structure such as blood vessels and damage to the cortical bone. Although most leaks are inconsequential [3], they all expose patients to serious risks. Extravasation of bone cement can lead to pulmonary cement embolism [4-8] and nerve root [9-11] as well as spinal cord compression $[12,13]$.

It has been previously documented that the cement viscosity is an important determinant of cement leakage and filling patterns in vertebral body augmentation, and the risk of cement leakage is reduced at high cement viscosity injection $[14,15]$. During polymerization of commonly used polymethylmethacrylate (PMMA) cements, the viscosity steadily increases over time. Hence, the cement should be injected at the latest time point possible in order to reduce the risk of leakage and extravasation. Consequently, the practitioner aiming for cement injection at high viscosity must wait for the cement viscosity to increase sufficiently, and this in turn reduces the total amount of working time of the cement. Waiting and working times for vertebroplasty cements according to the instructions for use at around $22^{\circ} \mathrm{C}$ are listed in the range from 2 to $7 \mathrm{~min}$ and 5 to $12 \mathrm{~min}$, respectively [16]. Depending upon the injection instrument, the force required for the delivery of high viscosity cement may exceed the human physical limits [14]. A method to overcome this problem is the use of cement delivery devices which are capable of generating high pressure. A main drawback in using such high pressure systems is the loss of the tactile feedback as opposed to simple syringe systems. A surgeon survey showed that the majority prefers 
simple syringe systems, which give the surgeon a direct force feedback during injection and therefore a very good tactile feeling for the procedure. Required working time mentioned by the surgeons was around $15 \mathrm{~min}$. Another potentially inconvenient effect of high viscosity cement could be in little interdigitation between cement and trabecular bone which compromise the mechanical strength of the reinforced vertebral body [13].

In order to address the reduction in waiting time, sufficient working time, and the usability of syringe systems, the development of a new PMMA vertebroplasty cement was undertaken. The goals of that development were to achieve a high initial viscosity just after mixing, diminish waiting time, and increase working time. These properties would allow the surgeon to begin injection immediately after cement preparation and continue with the procedure without waiting for the cement to reach a minimum viscosity level. As measure for the main goal, having a ready to use cement, the cement was investigated in vitro concerning cement leakage when injected directly after preparation. An experimental benchmark model, developed by Baroud et al. [15], was utilized to estimate both the leakage and intraosseous filling behavior of the new developed PMMA cement. Because the nature of the polymerization reaction is a radical reaction, it is accelerated at higher temperatures. In the body $\left(37^{\circ} \mathrm{C}\right)$ the cement cures faster than in ambient temperature, which has to be kept in mind during such experimentation and vertebroplasty procedures [17].

The transferability of the results from the study by Baroud et al. [15] to the clinical usage is limited. First, surgeons do not have an objective method to determine cement viscosity as measured in the study by Baroud et al. Second, the study did not incorporate medical grade products. Finally, the experiments were performed at room temperature, without any simulation of body temperature, which is not consistent with the clinical situation. In order to overcome those deficiencies and therefore to increase the transferability of the results for practitioners, we repeated this study with the alterations of using medical grade vertebroplasty cements, instruments as used in the clinical setting, and by simulating the human body with $37^{\circ} \mathrm{C}$ water bath. Additionally, the cement viscosity was determined using a viscometer specially designed for PMMA vertebroplasty cements (Viscosafe Viscometer, Synthes GmbH, Oberdorf, Switzerland).

In order to verify the main finding of Baroud et al. [15] that the leakage rate is reduced with higher cement viscosity, a regular vertebroplasty cement was also investigated. Cement spreading was evaluated when starting injection at different cement viscosity points. To observe the cement viscosity and therefore the injection viscosity, the Viscosafe Viscometer as one component of the Vertecem Vertebroplasty System (Synthes GmbH, Oberdorf, Switzerland) was used.

The objective of this study was to examine the cement leakage and filling pattern of two vertebroplasty cement products using the established benchmark model of Baroud et al. [15] and adapting the mentioned alterations in order to come closer to the clinical situation.

\section{Materials and Methods}

A leakage model suggested in 2006 by Baroud et al. [15] was used for this study. This is an established experimental model of the leakage phenomenon in vertebral body augmentation with a path, simulating a blood vessel to facilitate and favor the forces underlying intravertebral cement flow and to provoke cement leakage. This benchmark model was utilized to estimate both the leakage and filling behavior of the two vertebroplasty cements. Cement I is a regular vertebroplasty cement (Vertecem Mixing Kit, Ref. 07.702.010, Synthes GmbH, Oberdorf, Switzerland) having a low initial viscosity after preparation. Cement II is a new development with the same intended use (Vertecem V+ Cement Kit, Ref. 07.702.016S, Synthes GmbH, Oberdorf, Switzerland) presenting a medium initial viscosity.

An important addition to the experimental protocol of Baroud et al. [15] was that the aluminum cylinders acting as vertebral body substitutes were placed in a water bath at 37 $\pm 1^{\circ} \mathrm{C}$, simulating human body temperature. In order to run the experiment using a $37^{\circ} \mathrm{C}$ water bath, a form-stable bone marrow simulant at $37^{\circ} \mathrm{C}$ had to be found.

All materials except for the vertebral body model are similar to those used in vertebroplasty surgery and are all available with regional restrictions for surgeons today. These materials include bone cement, syringes, needles, and viscometer for viscosity control.

Six experimental groups were evaluated. Five groups used Cement I, starting injection at a range of different viscosities. The sixth group consisted of Cement II, starting injection immediately subsequent to cement preparation.

\subsection{Preparation of the Leakage Model and the Bone Marrow} Substitute. The leakage model consists of a trabecular bone and bone marrow substitute. The trabecular bone substitute is made of Aluminum Oxide foams cut to shape, drill holes applied as described in the study by Baroud et al. [15].

The following steps were followed to prepare a starch mixture which is form stable at $37^{\circ} \mathrm{C}$ as bone marrow substitute: cornstarch powder (Maizena, Knorr AG, Thayngen, Switzerland) and cold water were mixed by a ratio of $1: 3$ by stirring thoroughly at room temperature until a uniform and homogeneous milk-like appearance was achieved. Next, the Aluminum foam was soaked into the mixture and the mixture was heated using medium heat while stirring constantly in the same direction. The mixture is stirred and heated until it begins to get thick and boil. Then stirring was stopped and the mixture was left on heat for 1-2 minutes before heating was stopped and the foam was removed. After the mixture cooled, the foam sample was placed in a refrigerator for 1 hour. Each sample was weighted before and after being filled with the starch mixture, assuring that at least $95 \%$ of the voids of the porous foams were filled [18]. The final preparation step of the leakage model consisted of attaching a thin layer of around $3 \mathrm{~mm}$ in thickness of acrylic cement (DP-Pour, DenPlus Inc., Montreal, QC) to give the model a hard shell. This thin layer was intended to act as a simulation of the cortical shell of the vertebral body. 
2.2. Cement Preparation and Hardening Behaviour. All cements used for injection experiments were medical grade polymethylmethacrylate cements. Cement I is a regular vertebroplasty cement (Vertecem Mixing Kit, Ref. 07.702.010, LOT 043R/0834, Synthes GmbH, Oberdorf, Switzerland) having a low initial viscosity after preparation. Cement II is a new development with the same intended use (Vertecem V+ Cement Kit, Ref. 07.702.016S, LOT 4191, Synthes $\mathrm{GmbH}$, Oberdorf, Switzerland) presenting a medium initial viscosity. The cements were prepared according to the manufacturer's instructions using a closed mixing device. The time after starting cement preparation was recorded using a stopwatch, started at the same moment as adding the liquid to the powder. $9 \mathrm{~mL}$ of the prepared cement was transferred using a luer-luer coupling adapter into three $3 \mathrm{~mL}$ syringes (Viscosafe Injection Kit, Ref. 07.702.210, Synthes $\mathrm{GmbH}$, Oberdorf, Switzerland) for injection and viscosity measurement. The first two syringes were used for cement injection, and the third syringe was submitted for viscosity measurement using a viscometer (Viscosafe Viscometer, Anton Paar, Graz, Austria, SN 80215110 REF. 03.702.010) [10] which was kept at $22 \pm 1^{\circ} \mathrm{C}$. The viscometer records real viscosity every $5 \mathrm{~s}$ directly to a PC using the corresponding software (RHEOPLUS/32 Multi 128 V2.66, Anton Paar, Graz, Austria).

2.3. Cement Injection Procedure. To perform injection tests, an 8 Ga Jamshidi biopsy needle (Angiotech Medical Device Technologies, Gainesville, USA) with a length of $150 \mathrm{~mm}$ was inserted into the injection channel as defined for the benchmark model. Further details, including exact needle placement and leakage model dimensions, can be found in the study by Baroud et al. [15]. 30 min prior to and during cement injection, the aluminium foam filled with starch solution and equipped with the needle was placed in a $37^{\circ} \mathrm{C}$ water bath to reach thermal equilibrium.

In order to investigate the cement spreading and leakage observed for different cement viscosities, Cement I was used. For injection, the $3 \mathrm{~mL}$ syringe filled with cement was attached to the $8 \mathrm{Ga}$ Jamshidi biopsy needles and mounted on a universal testing machine (MTS MiniBionix 858, MTS, 14000 Technology Drive Eden Prairie, MN, USA). The starting points of cement injection into the vertebral body substitute were determined by reaching a predefined viscosity threshold as measured in real time by the viscometer. Predefined viscosities for start injection were $10,50,100,200$, and $400 \mathrm{~Pa}^{*} \mathrm{~s}$. A total of $6 \mathrm{~mL}$ of cement was injected using a two-step injection of two $3 \mathrm{~mL}$ syringes. The injection rate was $3.5 \mathrm{~mL} / \mathrm{min}$.

Cement II was injected directly after transferring the cement to the syringes and mounting on the testing machine. Injection was started $3 \mathrm{~min}$ after the start of mixing, using the same cross-head speed of $3.5 \mathrm{~mL} / \mathrm{mm}$. For all cement groups, the time elapsed for changing from the first to the second syringe was always $90 \mathrm{~s}$.

During cement injection, the leakage model was observed for cement leakage from both sides of the model. Time after start mixing when the first leakage was observed defines the leaked time. After the entire cement injection procedure ended, the leaked cement was collected and weighted, defining the leaked mass. Afterwards, the injected foam was removed from the water bath and left at room temperature for 2 days to assure that the cement was totally cured. For the five groups using Cement I, five repeats were done. Seven repeats were performed using Cement II.

2.4. Analysis of Cement Spreading. To evaluate the filling of the leakage models, each specimen was cut normal to the specimen axis along the injection pathway, into two halves, using a water-cooled diamond saw. Then each half was washed with hot water to dissolve the starch solution. For both halves of the same specimen, images were taken, digitized and analyzed for eccentricity and averaged for the same specimens as described in detail in the study by Mohamed et al. [18]. Briefly, eccentricity is defined as the eccentricity of an ellipse having the same second moment of area as the filled configuration. The more uniform and circular the filled pattern is, the less the eccentricity value will be. For example, in a straight line the eccentricity is one, and for a circle it is zero.

2.5. Statistical Analysis. The measured endpoints were the eccentricity and mass of leaked cement (leaked amount/g) collected from the water bath at the end of each experiment. The influence of the material composition of Cement II and of the initial viscosity values of Cement I (fixed independent factors) on the leaked mass and eccentricity (dependent parameters) were statistically analyzed. Overall statistical analysis on the resulting six material groups was performed using univariate ANOVA. Because of the significant differences received from ANOVA $(P<.006)$, multiple post hoc comparisons were done by performing Tukey HSD test. In all cases, a $P$ value of $\leq .05$ was used as significance limit. Statistical analyses were performed using SPSS software version 15.0.

\section{Results}

Hardening behaviour of the cements characterized at $22^{\circ} \mathrm{C}$ by the real viscosity as function of time after starting cement preparation are shown as illustration in Figure 1. Figure 2 shows representative images of the leakage models after cutting, as submitted for quantitative eccentricity analysis. Qualitatively, high leakage mass was observed for Cement I when injected at low viscosity levels (e.g., $10 \mathrm{~Pa}^{*}$ s) resulting in cement distribution mainly along the path of least resistance, the predrilled holes. A more uniform filling could be obtained using higher injection viscosities up to $400 \mathrm{~Pa}^{*} \mathrm{~s}$ for Cement I. For Cement II the qualitative filling was more uniform (Figure 2) than all Cement I groups, even though it was injected immediately after cement preparation.

The results of leaked cement mass and leaked time are shown in Figure 3, presenting the mean \pm standard deviation for the six groups investigated.

As received from ANOVA, the leaked mass in Cement I groups decreased with the increase of the starting viscosity from 10 to $400 \mathrm{~Pa}^{*}$ s. $2.56 \pm 0.98 \mathrm{~g}$ of Cement I leaked when the cement was injected at an initial viscosity of 


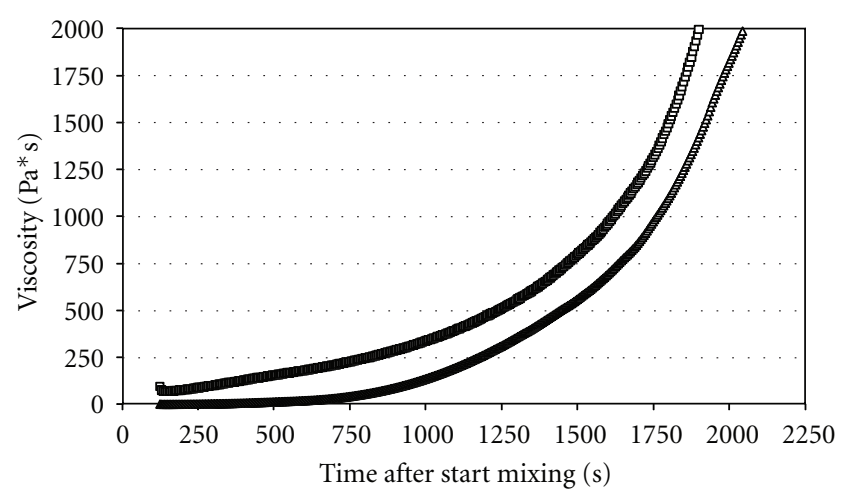

$\triangle$ Cement I

- Cement II

Figure 1: Hardening behavior at $22^{\circ} \mathrm{C}$ presented on the cement viscosity as function of the time after start mixing for the cements investigated.

$10 \mathrm{~Pa}^{*}$ s. Delaying the injection resulted in reduction of the leaked cement mass. When injected at an initial viscosity of $400 \mathrm{~Pa}^{*}$ s, only $1.07 \pm 0.82 \mathrm{~g}$ leaked.

For Cement II only minimal leakage was observed. More specifically, the average leaked amount was $0.36 \pm 0.54 \mathrm{~g}$, and the absolute leaked mass was below $1 \mathrm{~g}$ for all tests performed. Furthermore, of the seven leakage models injected with Cement II, there have been 3 observations without leakage.

Due to the high scattering of the data, especially the data received from the Cement I groups, statistical difference was low in general. Significant differences in leaked mass could be obtained between Cement I group injected at $10 \mathrm{~Pa}^{*} \mathrm{~s}$ and the Cement II group injected directly after mixing $(P=$ $.003)$. With a $P$-value of $.084(.173)$ the difference between the Cement I groups $10 \mathrm{~Pa}^{*} \mathrm{~s}$ versus $400 \mathrm{~Pa}^{*} \mathrm{~s}\left(200 \mathrm{~Pa}^{*} \mathrm{~s}\right)$ in leaked mass showed a clear trend in reduced leakage for higher injection viscosity. Comparison between the Cement I group injected at $50 \mathrm{~Pa}^{*} \mathrm{~s}$ and the Cement II group showed also a clear trend in reduced leakage rate using Cement II with a $P$ value of.061 (Figure 3). All other pairs yield not significant results presenting $P$ values higher than .257 .

Uniformity of the filling patterns quantified by the eccentricity for Cement I groups have shown no statistically significant differences in uniformity with the increase in injection viscosity (Figure 4). Cement II revealed a relative low eccentricity (Figure 4), similar to the visual impression in Figure 2. Statistical evaluation of the eccentricity values received from the sixth group consisting of Cement II showed significantly lower eccentricity in comparison to the Cement I groups injected at 10,50 , and $100 \mathrm{~Pa}^{*}$ s, presenting a $P$ value of $.005, .006$, and .03 , respectively. Comparison of Cement II to the Cement I group injected at $200 \mathrm{~Pa}^{*} \mathrm{~s}$ resulted in a trend of reduced eccentricity for the Cement II $(P=.079)$ (Figure 4).

\section{Discussion}

The objective of this study was to examine the cement leakage and filling behavior of a newly developed Vertebroplasty cement (Vertecem V+, Synthes $\mathrm{GmbH}$ ) injected immediately after preparation and a commercially available Vertebroplasty cement (Vertecem, Synthes $\mathrm{GmbH}$ ) when injected at a range of different viscosities.

The methods used herein were based on the benchmark experimental model established by Baroud et al. in 2006 to estimate the cement leakage behavior and filling pattern [15].

The added values of the new investigations in comparison to study published [15] were using exclusively medical grade products, Viscosafe Viscometer, to control cement viscosity and simulating body temperature using $37^{\circ} \mathrm{C}$ water bath. This resulted in more clinical relevance of the data observed due to immediate transferability to the clinic.

As mentioned in the literature [15], the model used in this study was designed to favor leakage, representing a worst case cement injection. In particular, the transverse drill hole of $3 \mathrm{~mm}$ is relatively large when compared to the demonstrated diameter from vertebral veins of 0.5 to $2 \mathrm{~mm}$ [19]. Furthermore, the relatively thick nature of the starch makes it difficult to displace, thereby decreasing the uniformity of filling and increasing the risk of cement leakage. Additional details concerning the displacement of bone marrow substitute can be found in the studies by Baroud et al. [20, 21] and Bohner et al. [14].

Clinical observations and investigations $[17,22]$ showed less to no leakage using commercial vertebroplasty cements (Cement I: Vertecem, Synthes GmbH; Vertebroplasty, J\&J DePuy Inc.) at a start injection viscosity of $50 \mathrm{~Pa}^{*}$ s. Cement I injected at this viscosity level demonstrated high leakage mass in the study presented, thus confirming that the experimental model used here favors leakage. Leaked mass observed for Cement I using start injection viscosities below $400 \mathrm{~Pa}^{*} \mathrm{~s}$ were still higher and comparable to Cement II when using $400 \mathrm{~Pa}^{*}$ s. Biggest difference in leaked mass investigated for Cement I could be observed between $100 \mathrm{~Pa}^{*} \mathrm{~s}$ and $200 \mathrm{~Pa}^{*}$ s. This is in good agreement with a similar investigation using Cement I [23]. High scattering of the parameters could be due to the model design using the $37^{\circ} \mathrm{C}$ water bath. Due to the accelerated polymerization and the step-wise injection procedure, same parts of the cement revealed higher viscosity already while the other parts remain at lower viscosities.

Experimental results demonstrate clearly the theoretical finding from Bohner et al. [14] in reduced leakage at higher starting viscosity using the Cement I. Results received from the Cement II showed low leakage rates in the used model favoring leakage. Hence, Cement II, which is available on the market in 2010, could be defined as a vertebroplasty cement which is ready for injection after preparation. Cement II pronounces a working time of at least $15 \mathrm{~min}$ for the entire ambient temperature ranging from 19 to $27^{\circ} \mathrm{C}$ [24], and it is applicable by using simple syringes allowing tactile feedback [24]. As presented herein Cement II will reduce the waiting time of the physician, in turn reducing the risk of too early injection and therefore will increase the safety of the intervention.

To estimate the injection viscosity of Cement I leading to a similar leaked mass as Cement II, an extrapolation was performed (Figure 4). A start injection viscosity for Cement I 

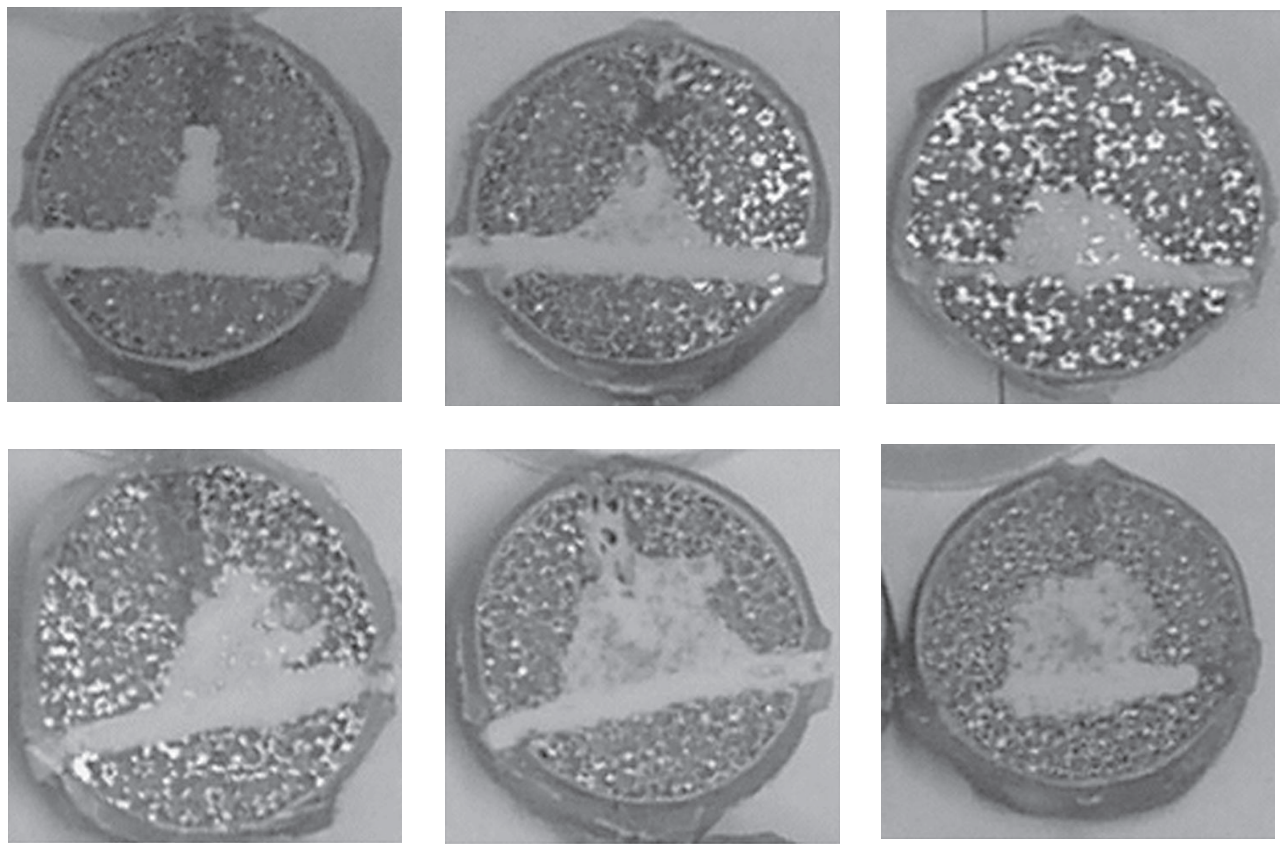

Figure 2: Pictures show representative images of the leakage models after cutting, as submitted for quantitative analysis. The upper line shows from left to right the result observed for Cement I at start injection viscosities of $10 \mathrm{~Pa}^{*} \mathrm{~s}, 50 \mathrm{~Pa}^{*} \mathrm{~s}$, and $100 \mathrm{~Pa}$ s. The lower line shows from left to right the result observed for Cement I at start injection viscosities of $200 \mathrm{~Pa}^{*} \mathrm{~s}, 400 \mathrm{~Pa}^{*}$ s, and Cement II injected immediately after preparation.

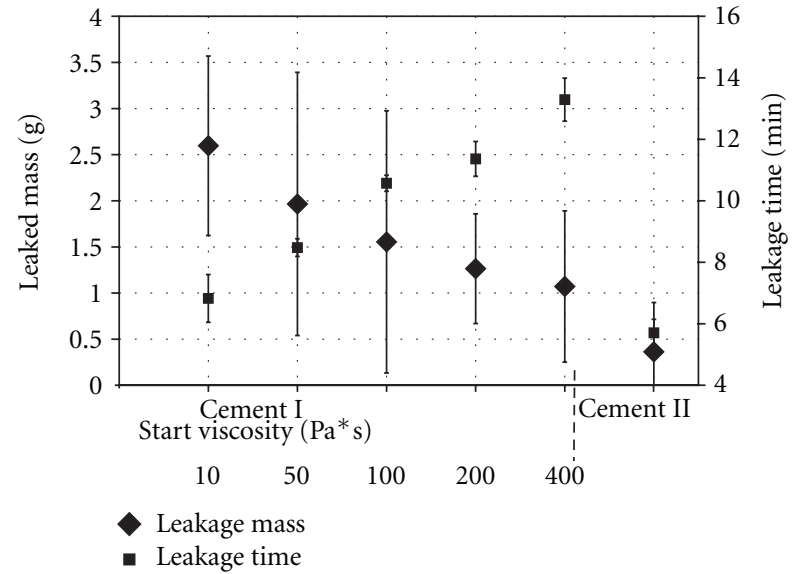

FIGURE 3: Leaked mass and leakage time presented in mean \pm standard deviation for the six groups investigated.

of around $600 \mathrm{~Pa}^{*}$ s yields the same amount of leaked mass as Cement II. In order to verify this phenomenological finding concerning leakage, the consistency of the cements at different viscosity levels was analyzed performing a visual inspection. The inspection of the cement consistency was done using extruding the cement out of a $1 \mathrm{~mL}$ syringe. Injection steps of $0.3 \mathrm{~mL}$ resulting in a cement spaghetti were extruded from the $1 \mathrm{~mL}$ syringe kept horizontally (Figure 5). Time for one injection step was tried to keep constant by around $2 \mathrm{sec}$. As measure for the cement consistency the lengthening of the cement spaghetti by gravitation force due to its own mass was used. Prior to each extrusion

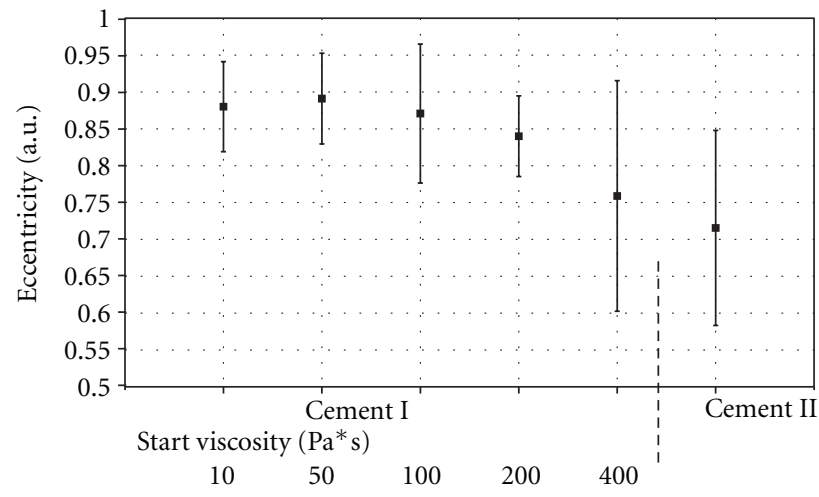

Figure 4: Average filling pattern estimated quantitatively by the eccentricity presented in mean \pm standard deviation for the six groups investigated.

the previous cement spaghetti was removed from the tip. Figure 5 shows representative trials of the comparison of $0.3 \mathrm{~mL}$ cement extruded out of the syringe for the six groups investigated in the study presented. The visual inspection of the consistency of Cement I at the mentioned viscosity (600 Pa*s) in comparison to Cement II immediately after preparation when the viscometer signaling a viscosity of around $80 \mathrm{~Pa}^{*}$ s showed a very good agreement in consistency (Figure 5).

In more detail, Cement I extruded having a viscosity of around $10 \mathrm{~Pa}^{*}$ s necked and lengthened right after starting extrusion followed by disrupter before the extrusion step could be finished. At a starting viscosity of around $50 \mathrm{~Pa}^{*} \mathrm{~s}$ 

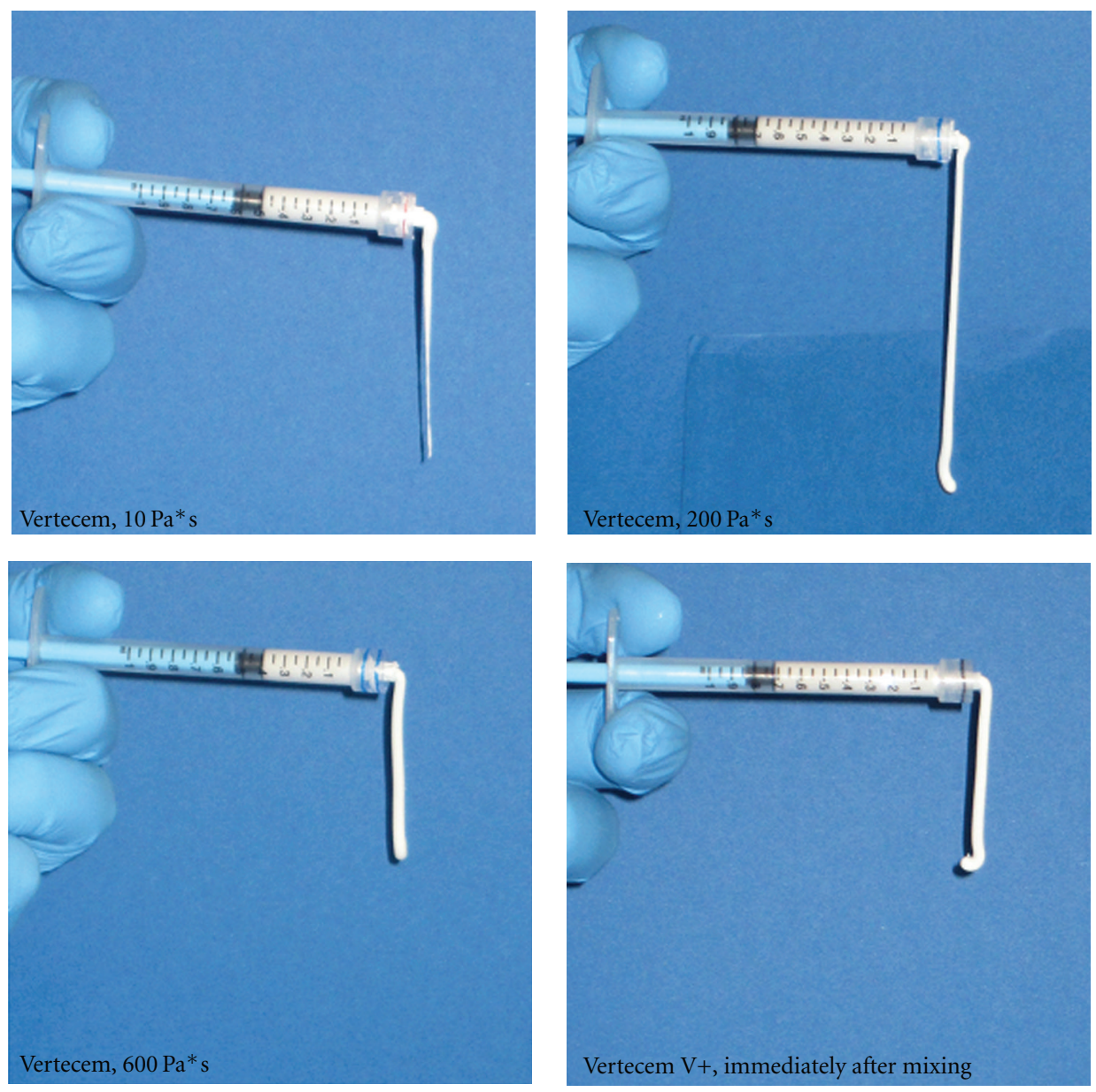

FIGURE 5: Pictures show representative trials of $0.3 \mathrm{~mL}$ cement extruded out of the syringe for the six groups investigated in the study presented.

the difference in behavior was visually in terms that the spaghetti stayed stable longer and disrupted at the end of the injection phase. Observation while injecting the cement having a viscosity of around $100 \mathrm{~Pa}^{*}$ s revealed a stable spaghetti for around $2 \mathrm{sec}$ before disruption was noticed. At $200 \mathrm{~Pa}^{*}$ s the $0.3 \mathrm{~mL}$ cement extruded demonstrated a lengthening without disruption after several seconds. Lengthening rate was reduced enormously using a cement viscosity of $400 \mathrm{~Pa}^{*}$ s, and no disruption could be observed after several seconds. As shown in Figure 5 lower line, the Cement I having a viscosity of $600 \mathrm{~Pa}^{*} \mathrm{~s}$ and the Cement II as observed just after preparation reveal a very similar behavior. Later was a stable cement spaghetti without any noticeable lengthening after around $20 \mathrm{sec}$. The initial Viscosity values at the start of extrusions in relation to the consistency observed for the groups indicates that measuring cement viscosity values as provided by the viscosafe viscometers measurement system are not suitable to compare different cement formulations on consistency; however, both are called PMMA cements. Thus, beside the real viscosity, there seem to be other physical phenomena that impact cement leakage and filling pattern such as elastic behavior of the cement. However, this will require further investigations and analysis of the rheological data. Results presented are restricted due to the experimental design of the used model. However, the model is biomechanically adequate [15] and no method is known which could be more suitable and closer to the clinical setting [25].

\section{Conclusion}

The investigation showed that with higher cement viscosity using Cement I (Vertecem Synthes GmbH) the leakage mass decreased and that the newly developed vertebroplasty cement (Vertecem V+ Cement Kit, Synthes GmbH) showed very low leakage mass in the used model favoring leakage when applied just after mixing. Later result leads to the claimed property of ready to use after preparation for the newly developed vertebroplasty cement (Vertecem V+). The added value of Cement II is that the waiting time for the physician will be shortened to zero min without compromising the safety for the procedure.

\section{Acknowledgment}

The authors kindly thank Professor Baroud and coworkers for performing the experiments by order of Synthes. 


\section{References}

[1] P. F. Heini, B. Walchli, and U. Berlemann, "Percutaneous transpedicular vertebroplasty with PMMA: operative technique and early results. A prospective study for the treatment of osteoporotic compression fractures," European Spine Journal, vol. 9, no. 5, pp. 445-450, 2000.

[2] F. Grados, C. Depriester, G. Cayrolle, N. Hardy, H. Deramond, and P. Fardellone, "Long-term observations of vertebral osteoporotic fractures treated by percutaneous vertebroplasty," Rheumatology, vol. 39, no. 12, pp. 1410-1414, 2000.

[3] P. Mousavi, S. Roth, J. Finkelstein, G. Cheung, and C. M. Whyne, "Volumetric quantification of cement leakage following percutaneous vertebroplasty in metastatic and osteoporotic vertebrae," Journal of Neurosurgery, vol. 99, no. 1, pp. 56-59, 2003.

[4] N. Aebli, J. Krebs, G. Davis, M. Walton, M. J. A. Williams, and J. C. Thesis, "Fat embolism and acute hypotension during vertebroplasty: an experimental study on sheep," Spine, vol. 27, no. 5, pp. 460-466, 2002.

[5] A. Gangi, S. Guth, J. P. Imbert, H. Marin, and J. L. Dietemann, "Percutaneous vertebroplasty: indications, technique, and results," Radiographics, vol. 23, no. 2, p. e10, 2003.

[6] D. H. Choe, E. M. Marom, K. Ahrar, M. T. Truong, and J. E. Madewell, "Pulmonary embolism of polymethyl-methacrylate during percutaneous vertebroplasty and kyphoplasty," American Journal of Roentgenology, vol. 183, no. 4, pp. 1097-1102, 2004.

[7] C. Duran, M. Sirvanci, M. Aydoğan, E. Ozturk, C. Ozturk, and C. Akman, "Pulmonary cement embolism: a complication of percutaneous vertebroplasty," Acta Radiologica, vol. 48, no. 8, pp. 854-859, 2007.

[8] H. L. Chen, C. S. Wong, S. T. Ho, F. L. Chang, C. H. Hsu, and C. T. Wu, "A lethal pulmonary embolism during percutaneous vertebroplasty," Anesthesia and Analgesia, vol. 95, no. 4, pp. 1060-1062, 2002.

[9] R. Schmidt, B. Cakir, T. Mattes, M. Wegener, W. Puhl, and M. Richter, "Cement leakage during the vertebroplasty: an underestimated problem?" European Spine Journal, vol. 14, no. 5, pp. 466-473, 2005.

[10] B. J. Lee, S. R. Lee, and T. Y. Yoo, "Paraplegia as a complication of percutaneous vertebroplasty with polymethylmethacrylate: a case study," Spine, vol. 27, no. 19, pp. E419422, 2002.

[11] J. S. Yeom, W. J. Kim, W. S. Choy, C. K. Lee, B. S. Chang, and J. W. Kang, "Leakage of cement in percutaneous transpedicular vertebroplasty for painful osteoporotic compression fractures," Journal of Bone and Joint Surgery, vol. 85, no. 1, pp. 83-89, 2003.

[12] A. W. Burton, L. D. Rhines, and E. Mendel, "Vertebroplasty and kyphoplasty: a comprehensive review," Neurosurgical Focus, vol. 18, no. 3, p. e1, 2005.

[13] P. A. Hulme, J. Krebs, S. J. Ferguson, and U. Berlemann, "Vertebroplasty and kyphoplasty: a systematic review of 69 clinical studies," Spine, vol. 31, no. 17, pp. 1983-2001, 2006.

[14] M. Bohner, B. Gasser, G. Baroud, and P. Heini, "Theoretical and experimental model to describe the injection of a polymethylmethacrylate cement into a porous structure," Biomaterials, vol. 24, no. 16, pp. 2721-2730, 2003.

[15] G. Baroud, M. Crookshank, and M. Bohner, "High viscosity cement significantly enhances uniformity of cement filling in vertebroplasty: an experimental model and study on cement leakage," Spine, vol. 31, no. 22, pp. 2562-2568, 2006.
[16] Product Info. KyphX HV-R, Kyphon Inc. Sunnyvale, CA 94089, USA; VertaplexTM, Stryker Instruments, Kalamazoo, MI 49001, USA; Spine-Fix, Synimed, 65502 Vic-en-Bigorre, France; SpineplexTM, Stryker Instruments, Kalamazoo, MI 49001, USA.

[17] A. Boger, K. D. Wheeler, B. Schenk, and P. F. Heini, "Clinical investigations of polymethylmethacrylate cement viscosity during vertebroplasty and related in vitro measurements," European Spine Journal, vol. 18, no. 9, pp. 1272-1278, 2009.

[18] R. Mohamed, C. Silbermann, A. Ahmari, M. Bohner, S. Becker, and G. Baroud, "Cement filling control and bone marrow removal in vertebral body augmentation by unipedicular aspiration technique: an experimental study using leakage model," Spine, vol. 35, no. 3, pp. 353-360, 2010.

[19] H. Frank and M. D. Netter, Atlas of Human Anatomy, Rittenhouse, 2nd edition, 2001.

[20] G. Baroud, R. Falk, M. Crookshank, S. Sponagel, and T. Steffen, "Experimental and theoretical investigation of directional permeability of human vertebral cancellous bone for cement infiltration," Journal of Biomechanics, vol. 37, no. 2, pp. 189196, 2004.

[21] G. Baroud, R. Falk, M. Crookshank, S. Sponagel, and T. Steffen, "Experimental and theoretical investigation of directional permeability of human vertebral cancellous bone for cement infiltration," Journal of Biomechanics, vol. 37, no. 11, p. 1802, 2004.

[22] Tancioni et al., "Cement Leakage observed during 3 year experience using the synthes vertebroplasty system," In press.

[23] M. Loeffel, S. J. Ferguson, L. P. Nolte, and J. H. Kowal, "Vertebroplasty: experimental characterization of polymethylmethacrylate bone cement spreading as a function of viscosity, bone porosity, and flow rate," Spine, vol. 33, no. 12, pp. 13521359, 2008.

[24] S. Deusser, C. Sattig, and A. Boger, "Rheological and curing behavior of medium-viscosity acrylic cement for cancellous bone augmentation," submitted to Journal of Biomedical Material Research.

[25] R. Rosiek, K. Wheeler, A. Boger et al., "Klinische Relevanz eines humanen Kadavermodells zur Beurteilung von Zementextravasation und -Ausbreitung bei Vertebroplastik," in Proceedings of the 4th Deutscher Wirbelsäulenkongress, München, Deutschland, 2009. 

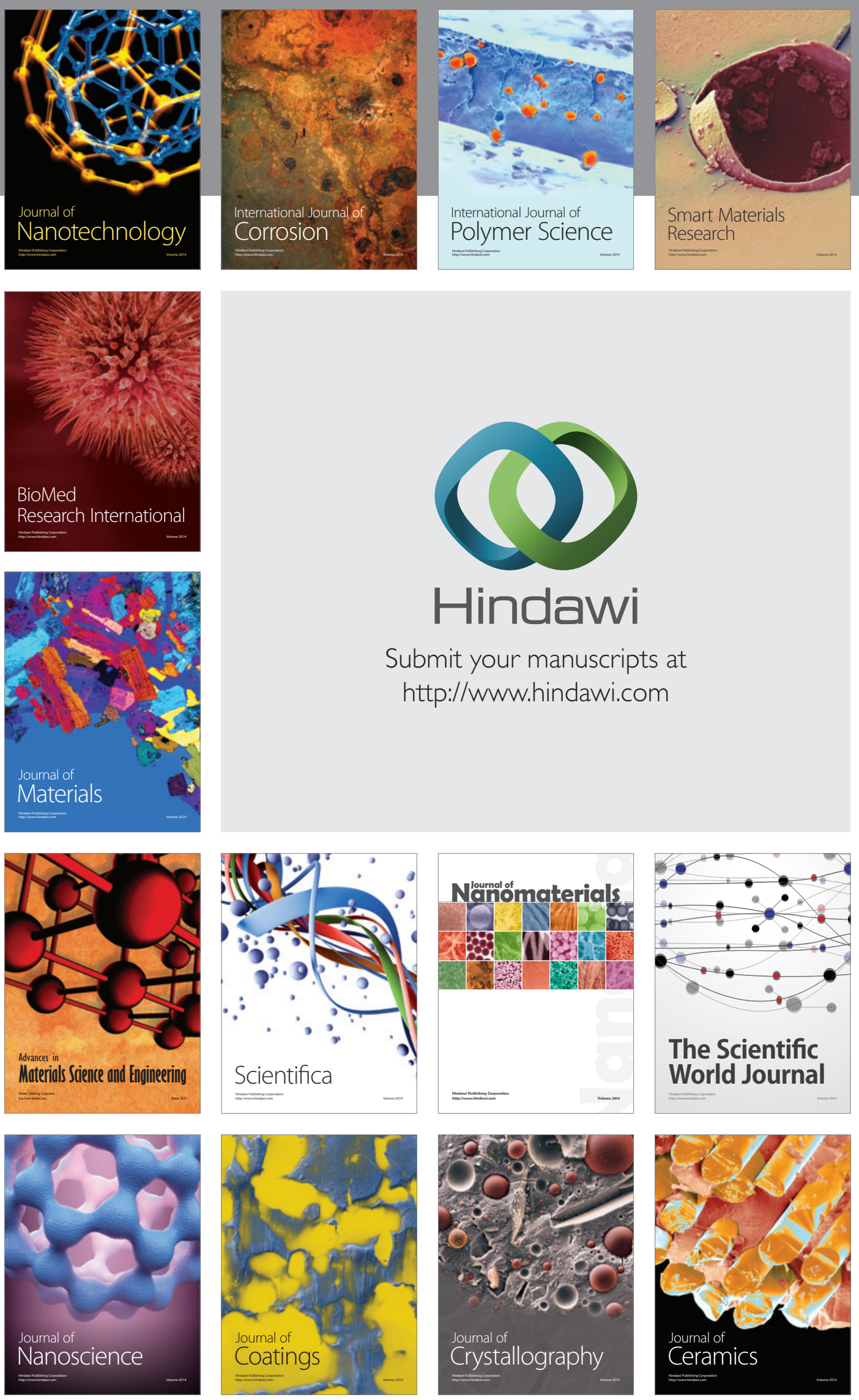

The Scientific World Journal

Submit your manuscripts at

http://www.hindawi.com

\section{World Journal}

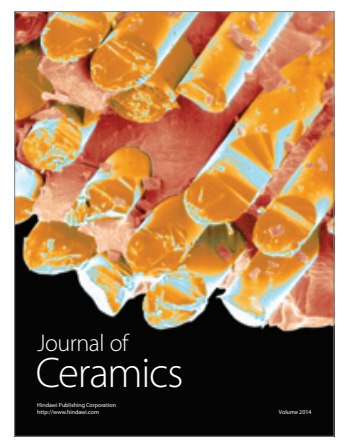

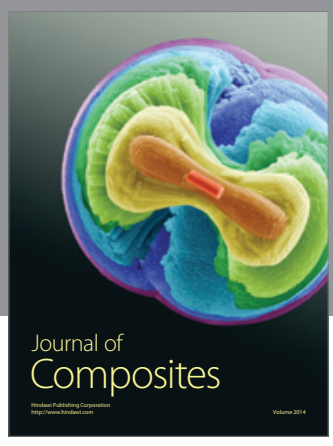
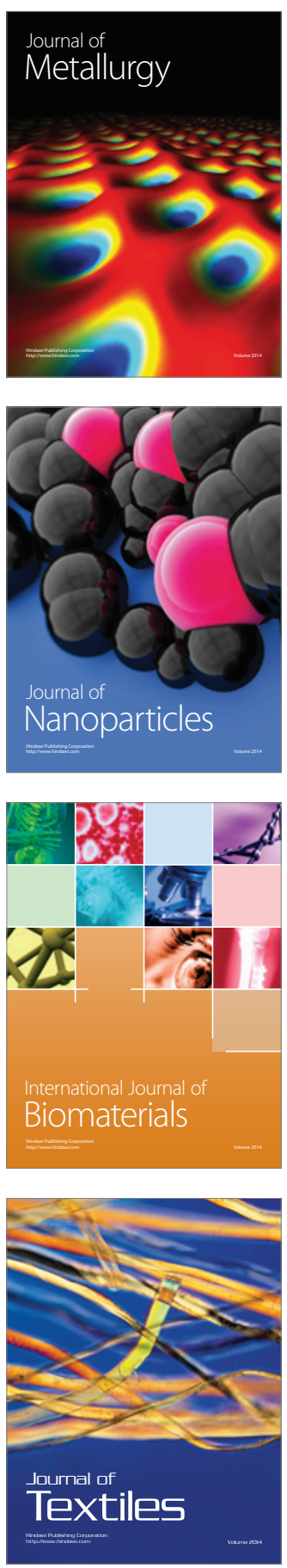